\title{
Power Line Communication Over Flexible Cables for Elevator Cabins
}

\author{
Motuma Abafogi ${ }^{1, ~ *}$, Bayram Akdemir ${ }^{1}$, Abdulfetah Abdela Shobole ${ }^{2}$ \\ ${ }^{1}$ Department of Electrical and Electronics Engineering, Konya Technical University, \\ Konya, Turkey \\ ${ }^{2}$ Department of Electrical and Electronics Engineering, Istanbul Sabahattin Zaim University, \\ Halkali Mahallesi, Istanbul, Turkey \\ e168121001013@ktun.edu.tr
}

\begin{abstract}
Narrowband power line communication has become a major focus of research with its applicability extending to residential facilities. This article examines data transmission over flexible AC power line cables stretching from the elevator control panel to the cabin. In previous studies, there has been no significant attempt to characterize the Power Line Communication (PLC) channel and noise of an elevator system. In this article, a vector network analyzer and mixed domain oscilloscope along with coupling devices are used to conduct power line channel noise and response measurements for an elevator system. The acquisition of channel transfer functions corresponding to both idle and moving states of the elevator is carried out for the $9 \mathrm{kHz}$ $500 \mathrm{kHz}$ frequency range. A mathematical model is provided for the average frequency response of the PLC channel. The main source of noise is the inverter that supplies power to the motor. The channel exhibits notable variations for frequencies less than $281 \mathrm{kHz}$ while staying relatively consistent for the $281 \mathrm{kHz}$ to $500 \mathrm{kHz}$ frequency range as the elevator changes from idle to moving state. Reed Solomon and convolutional codes along with interleaving to spread erroneous bits are utilized in this work to simulate an orthogonal frequency division multiplexing transceiver. By systematically selecting subcarrier frequencies that do not overlap with the harmonic noise components, simulation results have demonstrated reliable data recovery for signal-to-noise ratio greater than $4 \mathrm{~dB}$.
\end{abstract}

Index Terms-Power line communication; Elevator; Channel transfer function; Noise.

\section{INTRODUCTION}

Power line communication (PLC) uses existing infrastructure to facilitate data transmission over cables already established solely for power transmissions. PLC has become a promising candidate for smart grid applications with standards such as the European Committee for Electrotechnical Standardization (CENELEC) and the Federal Communications Commission (FCC) managing narrowband PLC that utilize frequencies below $500 \mathrm{kHz}$. Broadband PLC operates at higher frequencies, delivering very high data rates. Although PLC technology is appealing, establishing reliable transmission has been a difficult task due to the frequency selective fading and time-varying channel that is impaired by colored background and

Manuscript received 27 December, 2021; accepted 7 February, 2022. impulsive noise.

Many researchers have attempted to provide a mathematical model for the PLC channel and impulse response. There are two approaches to modelling the PLC channel: top-down, which attempts to determine a fitting mathematical model to channel response data gathered via extensive real-time measurements, and bottom-up, which relies on transmission line theory.

A mathematical PLC channel model described in [1] adopts a top-down approach that comprises a few parameters to express the complex channel transfer function corresponding to the PLC channel for the $500 \mathrm{kHz}$ to $20 \mathrm{MHz}$ frequency range. The model proposed in [2] further extends this model by putting forth ways to randomly generate PLC channel responses that are in agreement with the measured channels. Analysis of the characteristics of the PLC channel in residential areas in Brazil is presented in [3] for the frequency range of $1.7 \mathrm{MHz}-50 \mathrm{MHz}$ by employing extensive channel measurements. The access impedance modelling for Brazilian residences is provided in [4] for the $2 \mathrm{MHz}$ to $500 \mathrm{MHz}$ frequency range. Artificial bee colonybased modelling of indoor PLC channel measurements conducted in several cities in Turkey is presented in [5] for the $500 \mathrm{kHz}$ to $30 \mathrm{MHz}$ frequency range. Many bottom-up approach-based PLC channel generating models that use essential parameters such as topology, load impedances have been proposed in [6]-[9]. Similarly, in [10], the narrowband PLC channel simulations are discussed for numerous cable configurations, which are then validated using experimental results.

In [11], [12], performance evaluation for uncoded and low-density parity-check (LDPC) coded Orthogonal Frequency Division Multiplexing (OFDM) systems is examined for various indoor power line communication channel scenarios. Similarly, in [13], image transmission over a PLC channel using an LDPC coded OFDM technique is demonstrated. The simulation results indicate that employing LDPC coded OFDM displays a significant improvement in a reliable image transmission at a lower signal-to-noise ratio (SNR).

There are generally five major classes of PLC noise: colored background noise, narrowband noise, and periodic impulsive noise asynchronous to the mains frequency, 
which often remain stationary from a few seconds to several hours, as well as periodic impulsive noise synchronous to the mains frequency and asynchronous impulsive noise [14]-[16]. However, studies conducted in narrowband PLC have shown that the dominant components of PLC noise are periodic noise and cyclostationary noise [17]-[19].

A few parameters are employed to express narrowband PLC noise as a Gaussian process with periodic instantaneous variance [17]. The model attempts to adequately depict the temporal cyclic nature that dominates lower frequency ranges, but fails to incorporate the timevarying aspect of narrowband PLC noise. The model introduced in [18], [19] manages to take into account both the time and frequency variations.

An elevator system incorporates both control and power cables stretching throughout the entire floor of a building. These cables are subjected to severe environmental conditions due to elevator motion, noise, etc. often leading to incorrect or delayed data transmissions or even disconnection. Utilization of these power cables to deliver both data and power to the cabins aids in installing a less complex system with fewer cables, hence allowing for simpler and less costly installation, upgrades, and repairs. The PLC over the flexible AC power cable can also be integrated into smart buildings with an already established PLC network, thus granting remote access to the elevator. Residents can access the elevator and call it remotely, thus avoiding waste of time, especially in crowded buildings.

In [20], a simple half-duplex communication over elevator AC power lines is demonstrated using the frequency-shift keying modulation technique. The employed PLC modem uses a $72 \mathrm{kHz}$ carrier frequency with a baud rate of 9600 . The patented work mentioned in [21] describes a PLC system design for an elevator system. It provides a detailed design to facilitate data communication over elevator power lines. It demonstrates that the use of a PLC system eliminates the need for separate control wiring other than the elevator power line cables.

The feasibility of establishing data transmission over existing elevator power cables is discussed in [22]. According to the research, since there is already an existing power line cable that runs through the entire elevator hoistway, powerline communication can be achieved by superimposing the data to be transmitted over the $50 \mathrm{~Hz}$ or $60 \mathrm{~Hz}$ powerline. Hence, the role of communication cables such as Category 5 cable (CAT5) can be replaced, resulting in a simpler installation with fewer cables and a lower installation cost.

Several factors can affect the reliable transmission of data over the power lines of an elevator system, which include transient surges, noise from nearby electrical devices, radio frequency transmitters, etc. The commonly used surge protection devices for PLC modems include metal oxide varistor (MOV), protection diodes, gas discharge tubes, etc. Components such as common-mode chokes are frequently used to eliminate the unwanted common-mode signals induced by radio frequency transmitters. These protection elements remove the undesired signals, thus aiding in the transmission of a clean PLC signal over the frequency band of interest. For a narrowband PLC channel, a bandpass filter can help suppress high-frequency noise components outside the transmission frequency range and prevent them from reaching the internal circuitry of the PLC modem [23], [24].

This paper aims to provide new experimental findings regarding narrowband power line communication over flexible $\mathrm{AC}$ power lines that stretch from the elevator control panel to the cabin, which can be summarized as follows.

- Various studies are available on narrowband PLC noise characterizations derived from measurements taken in residential areas. Unfortunately, there have been no characterizations related to power line communication noise along the power line cables of an elevator system. This work provides the noise characteristics that are present along the elevator power lines by conducting several practical measurements using a Tektronix MDO3022 Mixed Domain Oscilloscope (MDO).

- There are several narrowband PLC channel response models derived from different sites such as residential areas, but none of these results include the PLC channel characteristics of elevator power line cables. This work provides the PLC channel characteristics of an elevator system obtained by using the Rohde and Schwarz ZVL13 Vector Network Analyzer (VNA) to perform practical channel measurements during both idle and moving elevator states.

- This paper also illustrates an OFDM-based transceiver simulation that adopts the acquired narrowband PLC noise and channel characteristics of elevator power line cables.

There is still ongoing research to formulate a standard model for PLC channel response and noise. Due to the absence of any significant research on characterizing the PLC channel response and noise for an elevator system, the results obtained in this research can be used as a basis for future studies aimed at formulating a standard model for an elevator PLC system.

The remainder of this article is organized into three major sections. The first section concisely describes some of the existing narrowband PLC technologies and the techniques involved in the simulation of a transceiver based on real channel measurements. The measurement setup, noise and channel characterization of the PLC channel are presented in the subsequent section. The last section provides a comparative analysis of the channel coding techniques used in designing an OFDM transceiver system.

\section{OFDM-BASED NARROWBAND PLC}

OFDM manages the available spectrum by applying several overlapping orthogonal subcarriers. The frequency selectivity fading and the presence of multipath propagation over the PLC channel render OFDM-based solutions invaluable for transmitting at higher data rates. A robust and efficient data recovery technique is imperative to establish reliable data transmissions over severe PLC channels.

There are numerous technologies capable of overcoming the harsh and noisy narrowband PLC channel, thus delivering reliable communications. Powerline Intelligent Metering Evolution (PRIME) is an OFDM-based narrowband PLC technology that uses a part of the 
frequency range of the CENELEC A band $(42 \mathrm{kHz}-$ $89 \mathrm{kHz}$ ). It offers a reliable and robust data transmission in different areas such as Automated Meter Reading or Automated Meter Management at a minimal cost [25]. G3PLC is another OFDM-based standard capable of accommodating faster and more reliable long-range data transmissions utilizing the $35 \mathrm{kHz}-91 \mathrm{kHz}$ frequency range [26].

As indicated in [27], the forward correction method used in the PRIME PLC is the convolutional code, while the G3 PLC employs the Reed Solomon code on top of the convolutional code. Comparative analysis of PRIME and G3-PLC revealed that the PRIME PLC is more prone to noise, while G3-PLC tends to withstand severe channel conditions. PRIME offers less complexity with higher data rates in a less hostile environment, while G3-PLC maintains reliable communication in harsh and noisy channel conditions [27], [28].

Elevator control panels and cabins can consist of different electrical devices from different manufacturers. Like any other PLC channel, the elevator PLC channel is also susceptible to noise and channel deterioration due to the nearby electrical devices. While designing a reliable data transmission, an efficient and robust channel coding is necessary to recover the transmitted data from the corrupted received data. This work simulates an OFDM-based transceiver that uses Reed Solomon and convolutional coding techniques. Interleaving is applied to the coded data to spread the possible occurrence of data corruption, thus facilitating a simpler recovery mechanism at the receiver. In this work, block interleaving (mentioned in [29]), which involves sequentially writing the input data and retrieving the output symbols, is employed. A simple illustration of a $5 \times 3$ block interleaver is provided in Fig. 1 .

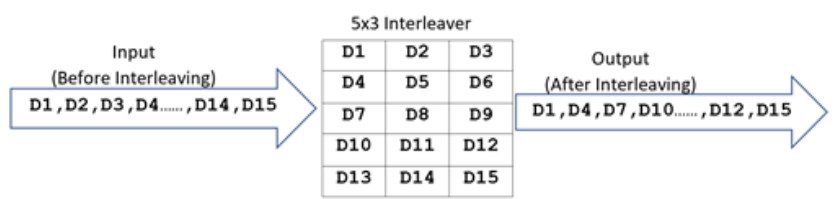

Fig. 1. Sequential writing and retrieval process of $5 \times 3$ block interleaver.

Decoding mechanisms that suffer from burst errors due to frequency selective fading channels benefit from frequency interleaving, which ensures the spreading of erroneous bits of adjacent subcarriers. In this work, a block interleaver, which has a block size equal to the number of bits per OFDM symbol $(\mathrm{N})$, is applied to perform interleaving per OFDM symbol.

It is arranged in a row-column matrix of size $\mathrm{R} \times \mathrm{C}=\mathrm{N}$, where $\mathrm{R}$ and $\mathrm{C}$ are the number of rows and columns, respectively. Interleaving is implemented by writing the input data into an $\mathrm{R} \times \mathrm{C}$ matrix row-wise and retrieving it column-wise, as indicated in Fig. 1.

The frequency-selective fading and time-varying PLC channel requires a robust channel estimation mechanism to recover the original data from erroneous data transmissions. The Comb-type Least Square (LS) channel estimation technique is used in this work to simulate the OFDM transceiver. For the $\mathrm{s}^{\text {th }}$ OFDM symbol and the $\mathrm{p}^{\text {th }}$ pilot location, the LS-based channel estimation at the corresponding pilot locations $\left(\mathrm{H}_{\mathrm{s}, \mathrm{p}}^{\mathrm{LS}}\right)$ is expressed in terms of the transmitted and received pilot symbols $\mathrm{X}_{\mathrm{s}, \mathrm{p}}$ and $\mathrm{Y}_{\mathrm{s}, \mathrm{p}}$ as in (1) [30], [31]

$$
\mathrm{H}_{\mathrm{s}, \mathrm{p}}^{\mathrm{LS}}=\frac{\mathrm{Y}_{\mathrm{s}, \mathrm{p}}}{\mathrm{X}_{\mathrm{s}, \mathrm{p}}} .
$$

Interpolation is employed to determine channel responses at locations other than the pilot subcarriers. The spline interpolation technique is implemented in this work.

\section{Channel Characterization}

The noise and channel characteristics of the $\mathrm{AC}$ power line that stretches from the elevator control panel to the elevator cabin are obtained using the measurement setup indicated in Figures 2 and 3. Measurements are carried out at the main office of EEM Elevator Control and Automation Systems in Konya, Turkey.

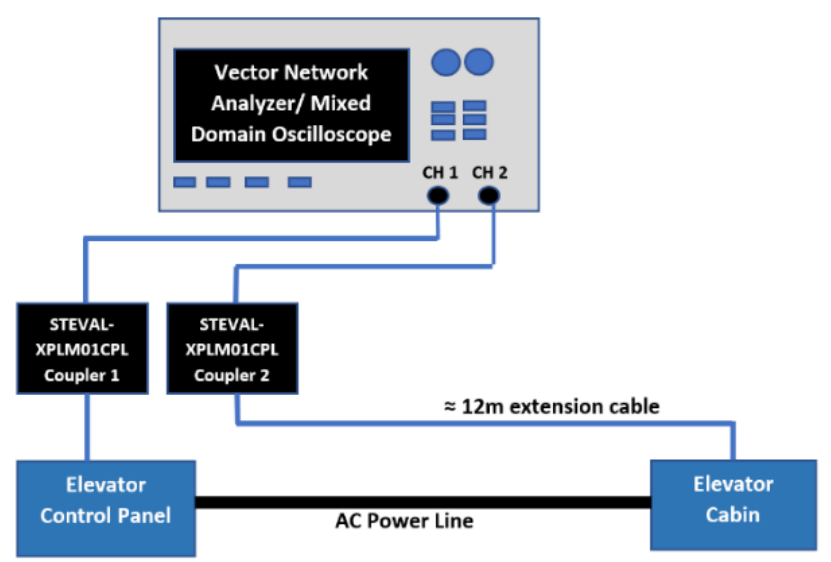

Fig. 2. Block diagram of the measurement setup.

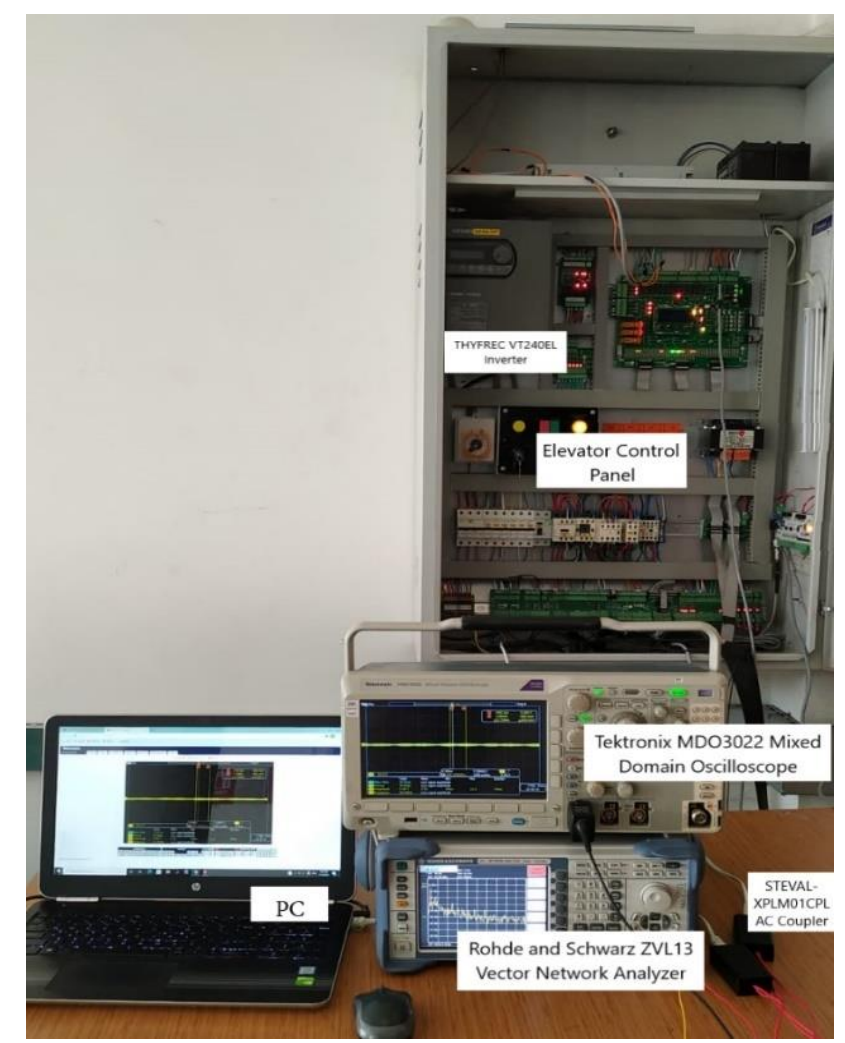

Fig. 3. Experimental Measurement setup. 
The channel transfer function for the AC power line is measured with Rohde and Schwarz ZVL13 Vector Network Analyzer (VNA) shown in Fig. 3. It has a $9 \mathrm{kHz}$ to $13.6 \mathrm{GHz}$ frequency range capable of measuring $\mathrm{S}$ parameters without reconnecting the device under test (DUT). The Tektronix MDO3022 Mixed Domain Oscilloscope (MDO) indicated in Fig. 3 is used to measure the PLC channel noise. It is an integrated oscilloscope that incorporates several functionalities, such as a spectrum analyzer and an arbitrary function generator. It is capable of sampling up to 5 Giga Samples/s with 10 Mega record length on every available channel. The VNA and MDO are connected to the power lines via two STEVALXPLM01CPL AC Coupling Circuits shown in Fig. 3 to ensure isolation from mains voltage.

\section{A. Channel Impulse and Frequency Response}

Several channel transfer function measurements were acquired with the help of Rohde and Schwarz ZVL13 Vector Network Analyzer (VNA) for both idle and moving elevator states for frequency values ranging from $9 \mathrm{kHz}$ to $500 \mathrm{kHz}$. The effect of the $12 \mathrm{~m}$ extension cable, as well as the two couplers depicted in Fig. 2, has been eliminated by a de-embedding process before processing the data. Figure 4 shows 50 separate measurements of the channel transfer function corresponding to the idle and moving states of the elevator. The channel experiences a sudden change in attenuation for frequencies less than $281 \mathrm{kHz}$ as the elevator starts moving while maintaining a relatively consistent attenuation for the $281 \mathrm{kHz}-500 \mathrm{kHz}$ frequency range. Consequently, data transmission in this study was conducted in the frequency range of $281 \mathrm{kHz}-488 \mathrm{kHz}$. Instantaneous measurements, as well as average channel responses for the idle and moving states of the elevator, have also been illustrated.

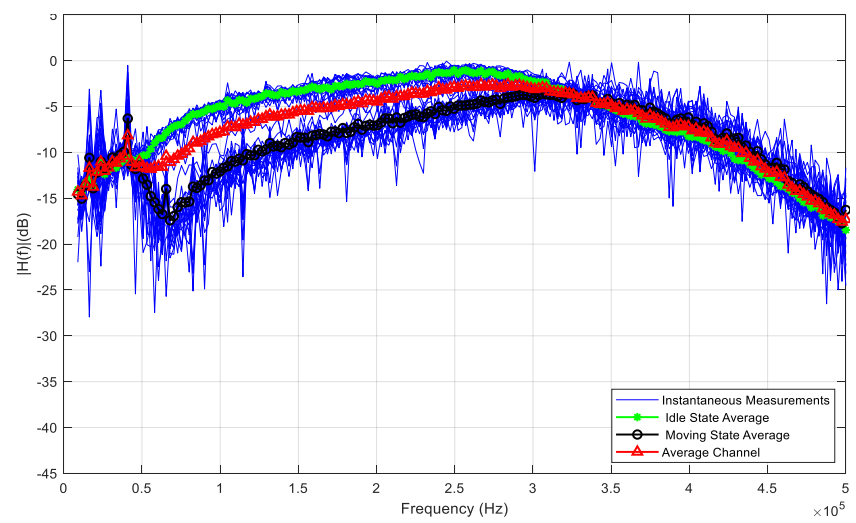

Fig. 4. Channel magnitude responses for idle and moving states of the elevator.

The propagation of the multipath signal for the power line channel stretching from the elevator control panel to the cabin can be expressed using the multipath signal propagation model depicted in [1], [32], [33]. Thus, the power line channel transfer function can be expressed as in (2)

$$
H(f)=\sum_{i=0}^{N} g_{i} \times e^{-\left(a_{0}+a_{1} f^{k}\right) d_{i}} e^{-j 2 \pi f\left(d_{i} / v_{p}\right)}
$$

The weight factor, length, and delay of the path $i$ are represented by the parameters $g_{i}, d_{i}$, and $d_{i} / v_{p}$, respectively. The parameter $\mathrm{N}$ denotes the number of dominant paths, while $\mathrm{a}_{0}, \mathrm{a}_{1}$, and $\mathrm{k}$ denote the attenuation factors corresponding to a particular power line channel. $v_{p}$ is the speed of propagation of a signal through the power line cable that is dependent on the dielectric constant $\varepsilon_{\mathrm{r}}$ of the insulating material. It has a value equal to $\mathrm{c} / \sqrt{ } \varepsilon_{\mathrm{r}}$ where $\mathrm{c}$ is the speed of light in a vacuum. The $\varepsilon_{\mathrm{r}}$ for the insulating material of the flexible cable in this elevator system is determined to be 4 . The parameter settings to model the power line channel of the elevator system are given in Table I.

TABLE I. CHANNEL RESPONSE MODELLING PARAMETERS.

\begin{tabular}{|c|c|c|}
\hline \multicolumn{3}{|c|}{ Path Parameters } \\
\multicolumn{2}{|c|}{$\left(\right.$ For $\left.N=\mathbf{2}, \boldsymbol{k}=\mathbf{0 . 7 9 3 6}, \boldsymbol{a}_{\mathbf{0}}=\mathbf{5 . 8 7 8} \times \mathbf{1 0}^{-\mathbf{4}} \mathbf{m}^{-\mathbf{1}}, \boldsymbol{a}_{\mathbf{1}}=\mathbf{- \mathbf { 2 . 8 8 9 }} \times \mathbf{1 0 ^ { - 8 }} \mathrm{s} / \mathbf{m}\right)$} \\
\hline $\mathbf{i}$ & $\mathbf{g}_{\mathbf{i}}$ & $\mathbf{d}_{\mathbf{i}}(\mathbf{m})$ \\
\hline 1 & 0.4322 & 150 \\
\hline 2 & -0.2602 & 450 \\
\hline
\end{tabular}

A two-path model representation of the average power line channel corresponding to the elevator system clearly shows very small variations from the measured channel magnitude and phase responses, as shown in Figures 5 and 6.

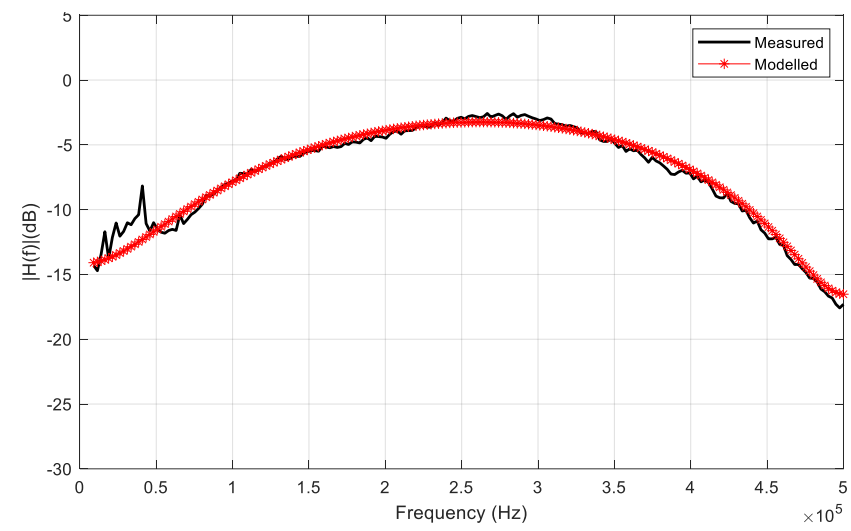

Fig. 5. Measured and modelled channel magnitude responses.

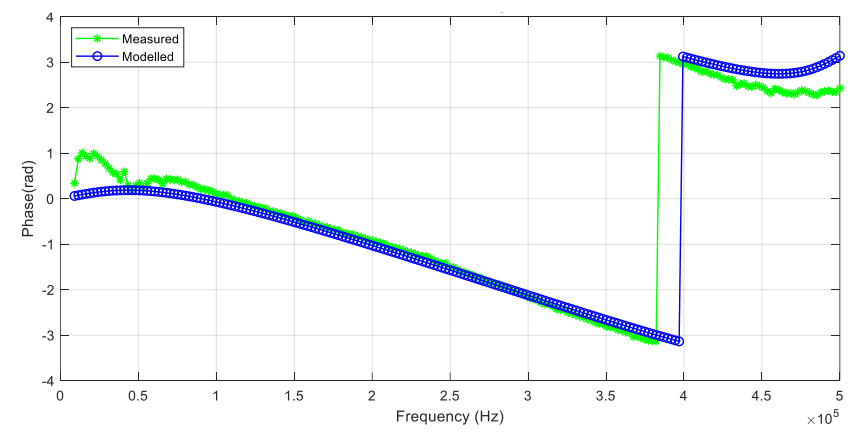

Fig. 6. Measured and modelled channel phase responses.

For the narrowband power line communication frequency range (up to $500 \mathrm{kHz}$ ), the impulse response is sampled at a period $\mathrm{T}_{\mathrm{s}}=1 \mu \mathrm{s}$. The peak values of the impulse response, which are indicative of the presence of multipath, help to determine the channel modelling parameters in Table I by adopting the steps described in [1]. Figure 7 shows the measured and modelled channel impulse responses.

The power delay profile (PDP) characterizes multipath channels by expressing the distribution of the power of the 
received signal over a multipath channel as a function of propagation delay. The PDP distribution corresponding to the average measured power line channel provided in Fig. 8 describes the power of the signal along each multipath with respect to the time delays. The formula used to determine the PDP from the measured channel impulse response is expressed as in (3) [34]

$$
P(t)=h(t) h^{*}(t)=|h(t)|^{2} .
$$

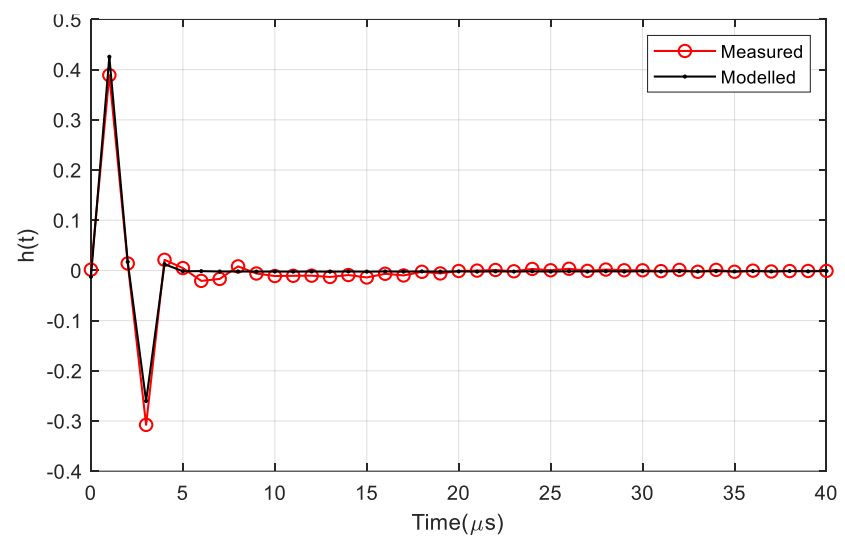

Fig. 7. Measured and modelled channel impulse responses.

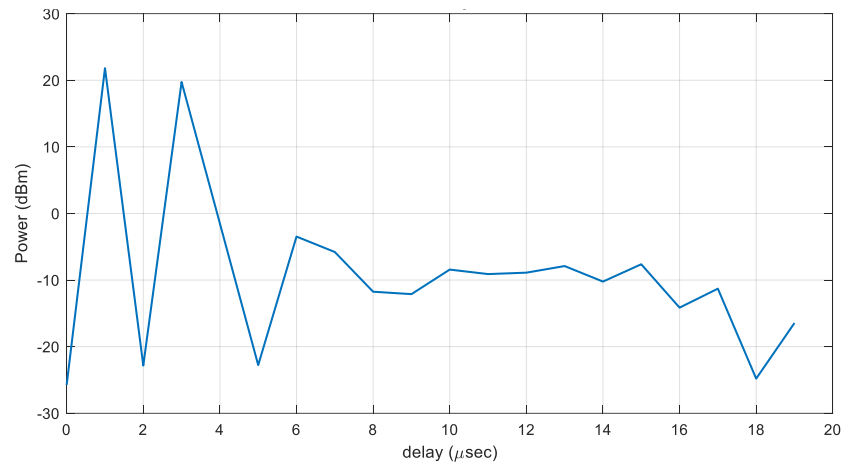

Fig. 8. Power delay profile.

The mean delay spread and the root mean square (RMS) delay spread of the channel can be expressed as in (4) and (5), respectively [23], [35]:

$$
\begin{gathered}
\tau_{\text {mean }}=\mathrm{T}_{\mathrm{s}} \times \frac{\sum_{\mathrm{k}=0}^{\mathrm{N}-1} \mathrm{k}\left|\mathrm{h}_{\mathrm{k}}\right|^{2}}{\sum_{\mathrm{k}=0}^{\mathrm{N}-1}\left|\mathrm{~h}_{\mathrm{k}}\right|^{2}}, \\
\tau_{\text {rms }}=T_{s} \times \sqrt{\frac{\sum_{k=0}^{N-1} k^{2}\left|h_{k}\right|^{2}}{\sum_{k=0}^{N-1}\left|h_{k}\right|^{2}}-\frac{\sum_{k=0}^{N-1} k\left|h_{k}\right|^{2}}{\sum_{k=0}^{N-1}\left|h_{k}\right|^{2}} .}
\end{gathered}
$$

The parameters $T_{s}, N$, and $h_{k}$ are the sampling time, the length of the impulse response, and the impulse response of the power line channel. For this channel, with $\mathrm{T}_{\mathrm{s}}=1 \mu \mathrm{s}$ and $\mathrm{N}=21$, the mean and RMS delay spread values are $\tau_{\text {mean }}=$ $1.8326 \mu$ s while $\tau_{\text {rms }}=1.2482 \mu \mathrm{s}$.

\section{B. Channel Noise}

Several noise samples were measured with the Tektronix MDO3022 Mixed Domain Oscilloscope (MDO). Power spectral density (psd) plots of 20 measured noise samples corresponding to both idle and moving states of the elevator provided in Fig. 9 were measured in peak detector mode.
During the elevator moving state, harmonic noise is introduced into the channel. The THYFREC VT240EL inverter shown in Fig. 3 above supplies power to the motor and it is set to a carrier frequency of $8 \mathrm{kHz}$, hence generating harmonic noise components with $8 \mathrm{kHz}$ fundamental frequency when the elevator is in motion.

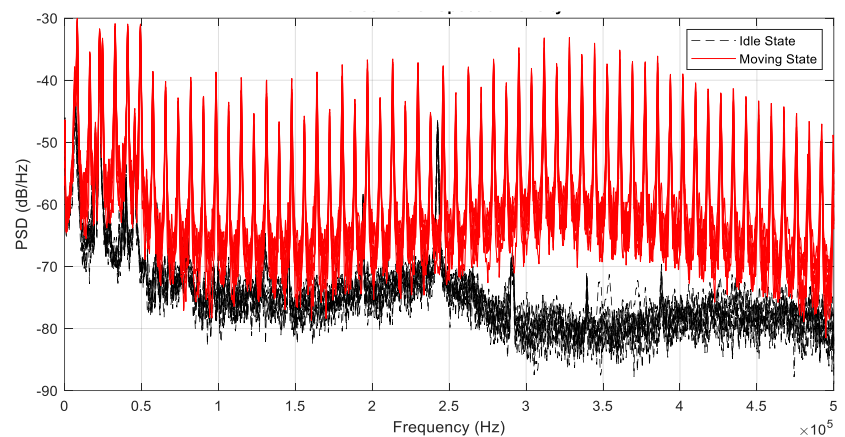

Fig. 9. Spectral density of noise power.

From the detailed description provided in the previous subsection, it is evident that for the $281 \mathrm{kHz}-488 \mathrm{kHz}$ frequency range the plc channel transfer function appears to be consistent regardless of the state of the elevator. By systematically selecting subcarrier frequencies that do not coincide with the harmonic noise components, a total of 77 subcarriers are utilized for data and pilot transmissions. The power spectral density of the transmitted signal having $133 \mathrm{~dB} \mu \mathrm{V}$ transmit power for the elevator moving state is estimated using the Welch's method, as shown in Fig. 10. The selection of the subcarrier frequencies mainly depends on the carrier frequency setting of the inverter. Different sets of subcarrier locations can be chosen to get more favorable results.

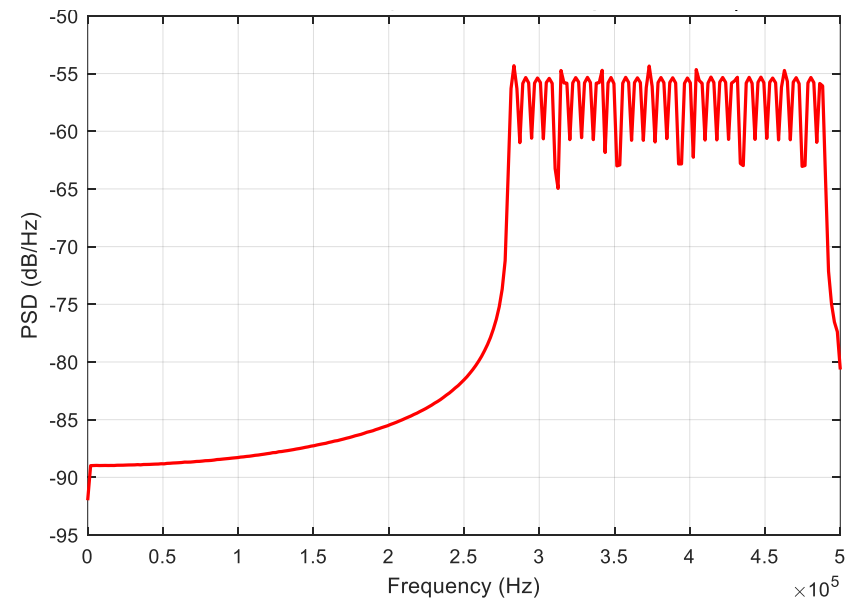

Fig. 10. Power spectral density of the transmitted signal at $133 \mathrm{~dB} \mu \mathrm{V}$.

\section{Channel Capacity}

The channel capacity is a parameter that corresponds to the theoretical upper bound of the bit transmission rate for a given channel. Due to the additive nature of the channel capacity over independent transmission channels, the total channel capacity is just the aggregate of the channel capacities of all the subcarriers. The capacity of the $\mathrm{k}^{\text {th }}$ subcarrier depends on both the subcarrier spacing $\Delta$ f and the signal-to-noise ratio of the $\mathrm{k}^{\text {th }}$ subcarrier $\left(\mathrm{SNR}_{\mathrm{k}}\right)$. For a given $\mathrm{N}$ number of subcarriers, the total capacity $\left(\mathrm{C}_{\text {tot }}\right)$ is 
expressed as in (6)

$$
C_{t o t}=\Delta f \times \sum_{k=1}^{N} \log _{2}\left(1+S N R_{k}\right) .
$$

For $133 \mathrm{~dB} \mu \mathrm{V}$ signal transmit power, independent channel capacities for the worst-case scenario of the channel (i.e., moving state of the elevator) with respect to $\mathrm{N}=77$ subcarrier frequencies mentioned in the previous section are given in Fig. 11. The total channel capacity is $145.44 \mathrm{kbps}$. Different results can be achieved by varying parameters such as the subcarrier spacing or by systematically increasing the number of subcarriers. Increasing the transmit power can also lead to an increase in the total channel capacity.

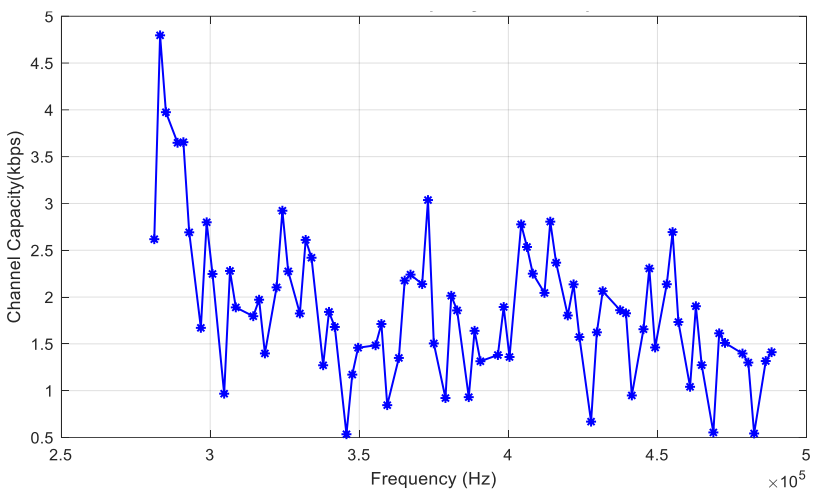

Fig. 11. Channel capacity versus frequency with an overall channel capacity of $145.44 \mathrm{kbps}$.

\section{OFDM TRANSCEIVER SIMULATION AND DISCUSSIONS}

Orthogonal frequency division multiplexing (OFDM) is a multi-carrier transmission technique that promotes the efficient utilization of the available bandwidth by dividing it into several orthogonal subcarriers. It is widely adopted in modern signal transmission systems for many reasons, including high spectral efficiency, robustness against frequency-selective fading channels, and resilience against multipath delay spread. Possible inter-symbol interferences (ISI) are mitigated by adding an appropriate length of cyclic prefix onto the transmitted signal.
The transmitter section of the OFDM transceiver illustrated in Fig. 12 is comprised of separate blocks such as channel coding, modulation, Inverse Fast Fourier Transform (IFFT), etc. This work utilizes concatenated Reed Solomon (RS) and Convolutional codes. The implemented RS coding has a block length of $n=255$ and message length of $\mathrm{k}=$ 239 , thus capable of correcting up to $t$ erroneous symbols where $2 \mathrm{t}=\mathrm{n}-\mathrm{k}$. A convolutional code with a $1 / 2$ code rate is used for the simulation along with the Viterbi decoder. The incorporation of an interleaver that spreads the transmitted data helps to mitigate burst errors due to frequency-selective fading affecting a few neighboring frequencies.

The coded input data is modulated with binary phase-shift keying (BPSK). One of the advantages of pilots in OFDM data transmission is that they can be adopted later by the receiver to characterize the nature of the transmission channel. In this work, 7 evenly spaced pilots are included. To effectively mitigate ISI, the cyclic prefix (CP) duration must be considerably larger than the delay spread highlighted in Section III-A above. A CP length of 32 is inserted before delivering the data to the PLC channel.

The average measured channel transfer function and measured noise for the moving state of the elevator (i.e., worst-case scenario) provided in Section III-A above are used for simulating the OFDM transceiver system. The channel estimation at the receiver is also accomplished using the Least Squares (LS) method described in the same subsection. The Bit Error Rate (BER) is determined by comparing the output of the Viterbi decoder followed by an RS decoder with the transmitted data.

The basic flow chart for the simulated OFDM transceiver system is given in Fig. 12.

A comparative analysis of the BER performance for different channel coding scheme combinations is given in Fig. 13. It is evident from the graph that interleaving reduces BER by spreading the erroneous bits. The convolutional code displays a considerable reduction in bit error rates when accompanied by an interleaver. Concatenated RS and convolutional codes ensure a reliable transmission for SNR values greater than $4 \mathrm{~dB}$.

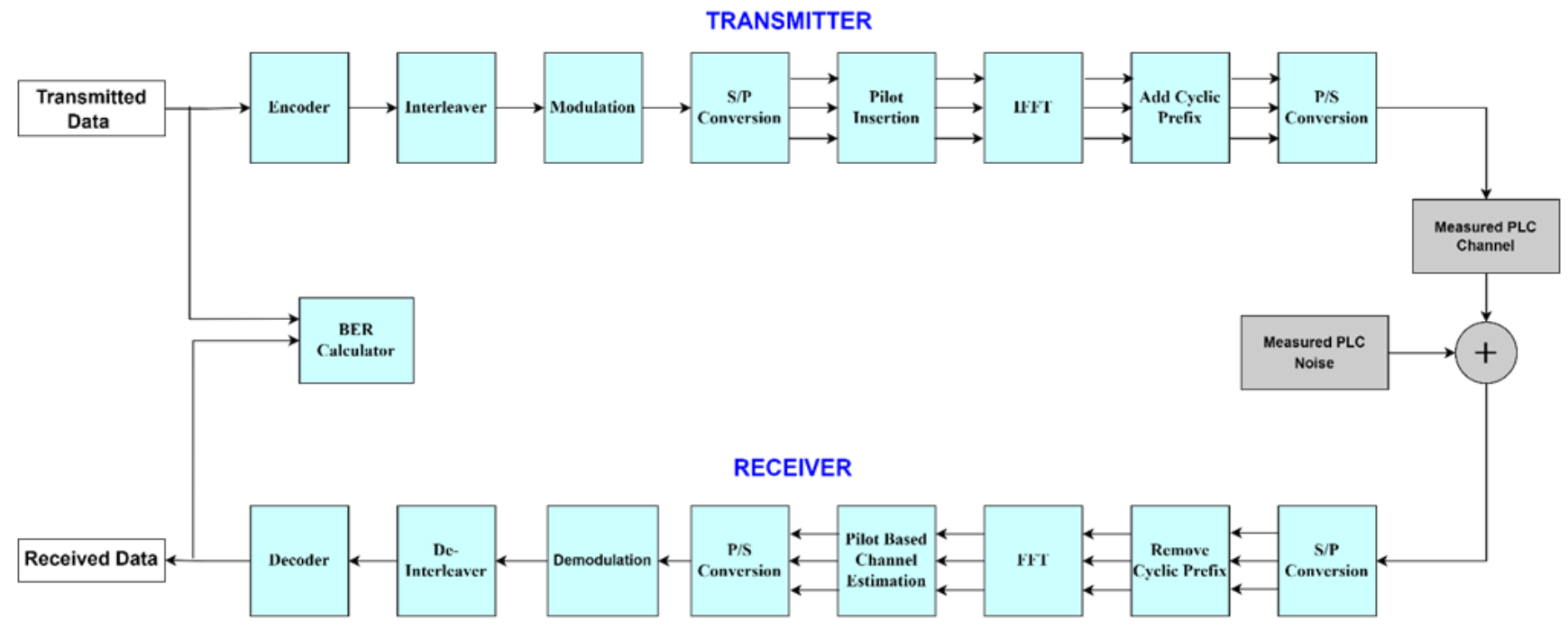

Fig. 12. OFDM transceiver block diagram. 
The simulation parameter settings are compiled in Table II.

TABLE II. OFDM TRANSCEIVER SIMULATION PARAMETERS.

\begin{tabular}{|c|c|}
\hline Simulation Parameters & Value \\
\hline Sampling Frequency & $1 \mathrm{MHz}$ \\
\hline FFT size & 512 \\
\hline Cyclic Prefix Length & 32 \\
\hline Subcarrier Spacing & $1.9531 \mathrm{kHz}$ \\
\hline Total Subcarriers & 77 \\
\hline Data Subcarriers & 70 \\
\hline Pilot Subcarriers & 7 \\
\hline Channel Coding & RS (255, 239), Convolutional \\
\hline Interleaving & Block interleaver \\
\hline Modulation Technique & BPSK \\
\hline Channel Estimation & LS \\
\hline Frequency Range & $281 \mathrm{kHz}-488 \mathrm{kHz}$ \\
\hline
\end{tabular}

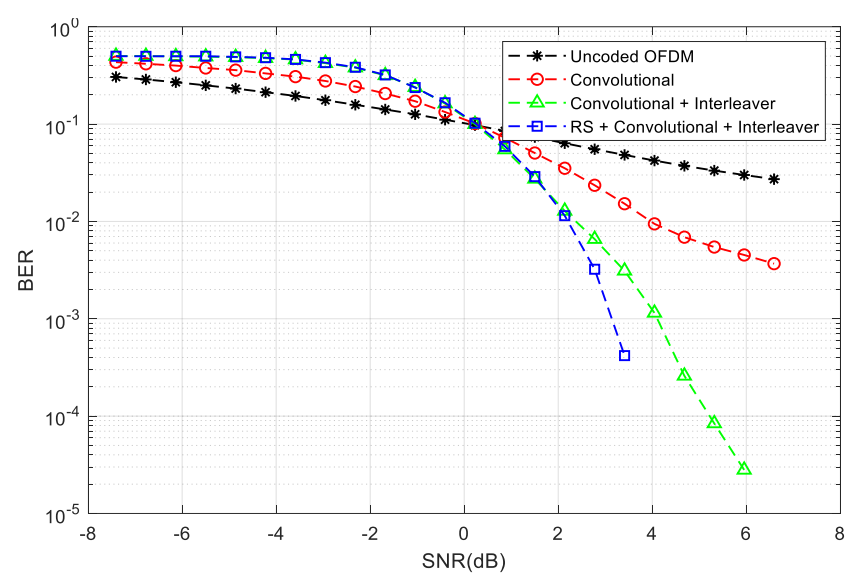

Fig. 13. BER versus SNR.

\section{CONClusions}

Data transmission over existing power lines has been a critical research area that has received widespread attention with new technologies emerging to facilitate reliable signal propagation. The main problems surrounding power line communication (PLC) technology are the varying channel response, as well as the noise introduced onto the received signal. One of the main interests of this work is characterizing the PLC channel for the AC power line stretching from the elevator control panel to the cabin, as well as characterizing the nature of the noise affecting data transmission. Video, voice, or data can be transmitted over $\mathrm{AC}$ power lines. The narrowband PLC frequency range is considered for analysis. This PLC channel has shown notable channel response variations for frequencies less than $281 \mathrm{kHz}$, while remaining relatively consistent for the $281 \mathrm{kHz}-500 \mathrm{kHz}$ frequency range. The main source of noise is determined to be the inverter that supplies power to the motor. It generates harmonic noise components with a fundamental frequency equal to the carrier frequency. This work has also managed to simulate an OFDM-based transceiver system by carefully selecting appropriate subcarrier frequencies. RS and Convolutional codes with interleaving are used to evaluate the BER performance of the system. As with any other PLC channel, elevator power lines are affected by the presence of disruptive signals from the surrounding. Additional protective circuit elements are included in PLC modems to effectively reject unwanted signals from reaching the circuit elements of PLC devices. Previous studies involved in characterizing the PLC channel and noise considered different areas like residential, institutions, etc. Unfortunately, no notable research has been done on an elevator PLC system. As there is no standard model for PLC channel and noise, the findings of this study can act as cornerstones for future experimentation that could aid standardizing the PLC channel and noise model of an elevator system. Consequently, further expansion of experimental scenarios can be employed, which may include the adoption of different types of elevator inverters with a varying number of floors. Future research can also include comparative analysis of different communication systems used for elevator systems. Simulation parameters of the transceiver system such as modulation technique, FFT length, number of subcarriers, etc. can also be altered to accommodate the transmission capacity of interest.

\section{CONFLICTS OF INTEREST}

The authors declare that they have no conflicts of interest.

\section{REFERENCES}

[1] M. Zimmermann and K. Dostert, "A multipath model for the powerline channel”, IEEE Trans. Commun., vol. 50, no. 4, pp. 553 559, 2002. DOI: 10.1109/26.996069.

[2] A. M. Tonello, F. Versolatto, B. Béjar, and S. Zazo, "A fitting algorithm for random modeling the PLC channel", IEEE Trans. Power Deliv., vol. 27, no. 3, pp. 1477-1484, 2012. DOI 10.1109/TPWRD.2012.2196714.

[3] T. R. Oliveira, A. A. M. Picorone, S. L. Netto, and M. V. Ribeiro, "Characterization of Brazilian in-home power line channels for data communication", Electr. Power Syst. Res., vol. 150, pp. 188-197, Sep. 2017. DOI: 10.1016/j.epsr.2017.05.011.

[4] L. G. da S. Costa et al., "Access impedance in Brazilian in-home, broadband and low-voltage electric power grids", Electr. Power Syst. Res., vol. 171, pp. 141-149, Jun. 2019. DOI 10.1016/j.epsr.2019.02.015.

[5] I. Develi, Y. Kabalci, and A. Basturk, "Artificial bee colony optimization for modelling of indoor PLC channels: A case study from Turkey", Electr. Power Syst. Res., vol. 127, pp. 73-79, Oct. 2015. DOI: 10.1016/j.epsr.2015.05.021.

[6] T. Esmailian, F. R. Kschischang, and P. G. Gulak, "In-building power lines as high-speed communication channels: Channel characterization and a test channel ensemble", Int. J. Commun. Syst., vol. 16, no. 5, pp. 381-400, 2003. DOI: 10.1002/dac.596.

[7] F. J. Cañete, J. A. Cortés, L. Díez, and J. T. Entrambasaguas, "A channel model proposal for indoor power line communications", IEEE Commun. Mag., vol. 49, no. 12, pp. 166-174, 2011. DOI: 10.1109/MCOM.2011.6094022.

[8] A. M. Tonello and F. Versolatto, "Bottom-up statistical PLC channel modeling-part I: Random topology model and efficient transfer function computation", IEEE Trans. Power Deliv., vol. 26, no. 2, pp. 891-898, 2011. DOI: 10.1109/TPWRD.2010.2096518.

[9] G. S. Lima and A. De Conti, "Bottom-up single-wire power line communication channel modeling considering dispersive soil characteristics", Electr. Power Syst. Res., vol. 165, pp. 35-44, Dec. 2018. DOI: 10.1016/j.epsr.2018.08.015.

[10] T. A. Papadopoulos, A. I. Chrysochos, and G. K. Papagiannis, "Narrowband power line communication: Medium voltage cable modeling and laboratory experimental results", Electr. Power Syst. Res., vol. 102, pp. 50-60, Sep. 2013. DOI: 10.1016/J.EPSR.2013.04.009.

[11] I. Develi and Y. Kabalci, "Analysis of the use of different decoding schemes in LDPC coded OFDM systems over indoor PLC channels", Elektronika ir Elektrotechnika, vol. 20, no. 10, pp. 76-79, 2014. DOI: 10.5755/j01.eee.20.10.8879.

[12] I. Develi and Y. Kabalci, "Highly reliable LDPC coded data transfer in home networks by using Canete's PLC channel model", Int. J. Electr. Power Energy Syst., vol. 62, pp. 912-918, Nov. 2014. DOI: 10.1016/j.ijepes.2014.05.051.

[13] I. Develi, Y. Kabalci, and A. Basturk, "Performance of LDPC coded 
image transmission over realistic PLC channels for smart grid applications", Int. J. Electr. Power Energy Syst., vol. 62, pp. 549-555, Nov. 2014. DOI: 10.1016/j.ijepes.2014.05.006.

[14] M. Zimmermann and K. Dostert, "Analysis and modeling of impulsive noise in broad-band powerline communications", IEEE Trans. Electromagn. Compat., vol. 44, no. 1, pp. 249-258, 2002. DOI: $10.1109 / 15.990732$.

[15] T. Bai et al., "Fifty years of noise modeling and mitigation in powerline communications", IEEE Commun. Surv. Tutorials, vol. 23, no. 1, pp. 41-69, 2021. DOI: 10.1109/COMST.2020.3033748.

[16] B. Masood et al., "Investigation of deterministic, statistical and parametric NB-PLC channel modeling techniques for advanced metering infrastructure", Energies, vol. 13, no. 12, p. 3098, 2020. DOI: $10.3390 /$ en 13123098 .

[17] M. Katayama, T. Yamazato, and H. Okada, "A mathematical model of noise in narrowband power line communication systems", IEEE $J$. Sel. Areas Commun., vol. 24, no. 7, pp. 1267-1276, 2006. DOI: 10.1109/JSAC.2006.874408.

[18] M. Nassar, A. Dabak, I. H. Kim, T. Pande, and B. L. Evans, "Cyclostationary noise modeling in narrowband powerline communication for Smart Grid applications", in Proc. of 2012 IEEE International Conference on Acoustics, Speech and Signal Processing $(I C A S S P), \quad 2012, \quad$ pp. 3089-3092. DOI 10.1109/ICASSP.2012.6288568.

[19] M. Nassar, J. Lin, Y. Mortazavi, A. Dabak, I. H. Kim, and B. L. Evans, "Local utility power line communications in the $3-500 \mathrm{kHz}$ band: Channel impairments, noise, and standards", IEEE Signal Process. Mag., vol. 29, no. 5, pp. 116-127, 2012. DOI: 10.1109/MSP.2012.2187038.

[20] N. S. Rajoba and M. M. Raste, "A Review on Hot Line communication for Lifts", Int. J. Eng. Trends Technol., vol. 67, no. 5, pp. 140-143, 2019. DOI: 10.14445/22315381/IJETT-V67I5P223.

[21] B. Zepke, R. Morgan, B. S. Dademade, and C. Slabinski, "Power line carrier used in elevator system", US 2003/0089558 A1, 2003.

[22] Heriyanto, M. Hamdani, and K. Wardhana, "Perancangan sistem komunikasi manajemen elevator menggunakan jaringan power line [Elevator management communication system design using power line network]", Sainstech, vol. 26, no. 2, pp. 68-76, 2016. DOI: 10.37277/stch.v26i2.512.

[23] L. Lampe, A. M. Tonello, and T. G. Swart, "Power Line Communications: Principles, Standards and Applications from Multimedia to Smart Grid, 2nd ed. John Wiley \& Sons, Ltd., 2016, p. 17, 108, 190, 254. DOI: 10.1002/9781118676684.

[24] L. G. da S. Costa, A. C. M. De Queiroz, B. Adebisi, V. L. R. da Costa, and M. V Ribeiro, "Coupling for power line communication: A survey”, Journal of Communication and Information Systems, vol. 32, no. 1, 2017. DOI: 10.14209/jcis.2017.2.

[25] A. Sanz, J. I. Garcia-Nicolás, P. Estopiñán, and S. Miguel, "PRIME from the definition to a SoC solution", in Proc. of 2009 IEEE International Symposium on Power Line Communications and Its Applications, $2009, \quad$ pp. 347-352. DOI 10.1109/ISPLC.2009.4913455.

[26] K. Razazian, M. Umari, A. Kamalizad, V. Loginov, and M. Navid, "G3-PLC specification for powerline communication: Overview, system simulation and field trial results", in Proc. of ISPLC2010, 2010, pp. 313-318. DOI: 10.1109/ISPLC.2010.5479881.

[27] M. Hoch, "Comparison of PLC G3 and PRIME", in Proc. of 2011 IEEE International Symposium on Power Line Communications and Its Applications, 2011, pp. 165-169. DOI 10.1109/ISPLC.2011.5764384.

[28] Z. Sadowski, "Comparison of PLC-PRIME and PLC-G3 protocols", in Proc. 2015 International School on Nonsinusoidal Currents and Compensation (ISNCC), 2015, pp. 1-6. DOI: 10.1109/ISNCC.2015.7174704.

[29] A. D. S. Jayalath and C. Tellambura, "Use of interleaving to reduce the peak-to-average power ratio of an OFDM signal", in Proc. of Globecom 'O0 - IEEE. Global Telecommunications Conference. Conference Record (Cat. No.00CH37137), 2000, pp. 82-86, vol. 1. DOI: 10.1109/GLOCOM.2000.891696.

[30] M. Bogdanović, "Frequency domain based LS channel estimation in OFDM based Power line communications", Automatika, vol. 55, no. 4, 2014. DOI: 10.7305/automatika.2014.12.639.

[31] P. Sure and C. M. Bhuma, "A survey on OFDM channel estimation techniques based on denoising strategies", Eng. Sci. Technol., an Int J., vol. 20, no. 2, pp. 629-636, 2017. DOI: 10.1016/j.jestch.2016.09.011.

[32] O. I. Arbab and M. Mirghani, "Power line communication channel modelling and system design", in Proc. of 2020 International Conference on Computer, Control, Electrical, and Electronics Engineering (ICCCEEE), 2021, pp. 1-4. DOI: 10.1109/ICCCEEE49695.2021.9429567.

[33] B. Masood, S. Guobing, R. A. Naqvi, M. B. Rasheed, J. Hou, and A U. Rehman, "Measurements and channel modeling of low and medium voltage NB-PLC networks for smart metering", IET Gener. Transm. Distrib., vol. 15, no. 2, pp. 321-338, 2021. DOI: $10.1049 / \operatorname{gtd} 2.12023$.

[34] G. J. M. Janssen, P. A. Stigter, and R. Prasad, "Wideband indoor channel measurements and BER analysis of frequency selective multipath channels at 2.4, 4.75, and $11.5 \mathrm{GHz}$, IEEE Trans. Commun., vol. 44, no. 10, pp. 1272-1288, 1996. DOI: $10.1109 / 26.539768$.

[35] A. A. M. Picorone, T. R. de Oliveira, R. Sampaio-Neto, M. Khosravy, and M. V. Ribeiro, "Channel characterization of low voltage electric power distribution networks for PLC applications based on measurement campaign", Int. J. Electr. Power Energy Syst., vol. 116 art. 105554, 2020. DOI: 10.1016/j.ijepes.2019.105554.

This article is an open access article distributed under the terms and conditions of the Creative Commons Attribution 4.0 (CC BY 4.0) license (http://creativecommons.org/licenses/by/4.0/). 